\title{
Nail Disease: Clinical Decisions among Portuguese Dermatologists and Family Physicians
}

\author{
Ana Filipe Monteiro $^{a}$ Rita Ramos Pinheiro ${ }^{b}$ Célia Galhardas ${ }^{b}$ \\ André Lencastre $^{b}$ \\ aDermatology Department, Hospital de Santarém EPE, Santarém, Portugal; bermatovenereology Department, \\ Hospital de Santo António dos Capuchos - Centro Hospitalar de Lisboa Central, Lisbon, Portugal
}

\section{Keywords}

Nail diseases · Onychomycosis · Surveys · Dermatologists ·

Family physicians

\begin{abstract}
Onychomycosis is one of the most common nail disorders and may be difficult to distinguish from other causes of nail dystrophy, based on clinical grounds alone. With this study, we aimed to describe the use of fungal testing by dermatologists and family physicians in their daily current practice, analyze their respective familiarity with nail disease diagnosis, and ultimately treatment decision-making by both groups. An online survey was distributed among Portuguese dermatologists, trainees, and family physicians by email. The survey focused on the diagnostic impression, use of diagnostic methods to confirm a fungal infection, and the subsequent assessment of treatment. One hundred fifty-one responses were obtained, 60 (39.7\%) from dermatologists and 91 (60.3\%) from family physicians; $98.3 \%$ of dermatologists mentioned usually requesting a fungal testing at their local institution or outside, while this percentage was $50.5 \%$ among family physicians $(p<0.001)$. Regarding the diagnosis, the median of correct diagnosis by the dermatologist
\end{abstract}

karger@karger.com www.karger.com/sad

(c) 2020 S. Karger AG, Basel

Karger!" group was higher (10/15) than the family physicians (6/15). Considering the treatment strategy, we observed that in the dermatologists' group it would result in unnecessary treatment in a median of 2 cases, while in the family physicians' group, in a median of 4 cases.

c) 2020 S. Karger AG, Basel

\section{Introduction}

Nail disorders are a common motive for consultation of both family physicians and dermatologists. Clinical evaluation can be challenging, and these disorders span a broad variety of diagnoses, which include infectious, inflammatory, and neoplastic causes. However, a firm familiarity with several common diagnoses and their appropriate evaluation can improve care of the patient with nail changes [1].

Onychomycosis is an especially common nail condition responsible for up to $50 \%$ of nail diseases. Across recently published epidemiology studies, the global prevalence of onychomycosis was estimated to be $5.5 \%$, falling within the range of previously reported estimates (2-8\%) [2]. 
Table 1. Characteristics of participants by specialty

\begin{tabular}{|c|c|c|}
\hline Characteristics & Dermatology & Family physician \\
\hline Average age, years (range) & $39.65(25-70)$ & $36.42(26-64)$ \\
\hline \multicolumn{3}{|l|}{ Gender } \\
\hline Male, $n(\%)$ & $24(40)$ & $28(30.8)$ \\
\hline Female, $n(\%)$ & $36(60)$ & $63(69.2)$ \\
\hline Total, $n$ & 60 & 91 \\
\hline \multicolumn{3}{|l|}{ Practice type } \\
\hline Private clinic exclusively, $n(\%)$ & $10(16.7)$ & $4(4.4)$ \\
\hline Public healthcare system exclusively, $n(\%)$ & $9(15)$ & $59(64.8)$ \\
\hline Both, $n(\%)$ & $15(25)$ & $3(3.3)$ \\
\hline Mainly private, $n(\%)$ & $1(1.7)$ & $0(0)$ \\
\hline Mainly public, $n(\%)$ & $25(41.7)$ & $25(27.5)$ \\
\hline Total, $n$ & 60 & 91 \\
\hline \multicolumn{3}{|l|}{ Request of mycology diagnostic tests } \\
\hline Request tests at their institution or outside, $n(\%)$ & $59(98.3)$ & $46(50.5)$ \\
\hline Do not request, $n(\%)$ & $1(1.7)$ & $45(45.5)$ \\
\hline Total, $n$ & 60 & 91 \\
\hline \multicolumn{3}{|l|}{ Access to mycology diagnostic tests } \\
\hline Culture at their institution, $n(\%)$ & $4(6.7)$ & $2(2.2)$ \\
\hline Culture + direct microscopy at their institution, $n(\%)$ & $42(70)$ & $2(2.2)$ \\
\hline Outside their institution, $n(\%)$ & $13(21.7)$ & $42(46.2)$ \\
\hline Do not have access, $n(\%)$ & $1(1.7)$ & $45(49.5)$ \\
\hline Total, $n$ & 60 & 91 \\
\hline
\end{tabular}

The clinical signs of onychomycosis are often difficult to distinguish from other causes of nail dystrophy [3]. Laboratory confirmation of a clinical diagnosis with identification of the causal organism should be obtained before starting treatment as the efficacy of oral and topical therapies can be species dependent [2-4]. Several methods can be used to confirm the diagnosis: direct microscopy using potassium hydroxide, periodic acid-Schiff staining of nail clippings, fungal culture, and molecular diagnostic methods such as PCR have recently been developed [1]. Diagnosing onychomycosis using more than 1 technique is a common practice among current onychomycosis studies. Potassium hydroxide and fungal culture are the most commonly used combination and is considered the current standard for diagnosing onychomycosis [2].With this study, we aimed to describe the use of fungal testing by dermatologists and family physicians in their daily current practice, analyze their respective familiarity with nail disease diagnosis, and ultimately treatment decision-making by both groups.

\section{Materials and Methods}

An online survey using Google Forms ${ }^{\circledR}$ was distributed among Portuguese dermatologists, trainees, and family physicians by email. Overall, the survey focused on the diagnostic impression, use of diagnostic methods to confirm a fungal infection, and the subsequent assessment of treatment. The first part of the survey contained questions regarding personal and professional data, such as access and use of mycology diagnostic techniques. A second part consisted of 15 select clinical cases that included cases of toe onychomycosis and nail dystrophy due to biomechanical causes (e.g., shoewear, podological, or gait alterations). Each clinical case displayed 2 to 3 high-quality photographs of patients' feet and toenails, and 2 questions, first regarding the diagnosis and the second regarding the participants' views on their workup/treatment strategy. A short text accompanied the clinical vignette, and responders were informed that no case had any signs or confirmation of fungal infection elsewhere on the skin. In preparation for the survey, the questionnaire was pretested on 10 volunteers (composed of 6 dermatologists and 4 family physicians).

The clinical cases were selected from the photo repository of the Dermatovenereology Department, Hospital de Santo António dos Capuchos, Lisbon. All cases had previously had appropriate diagnostic confirmation by clinical assessment and follow-up, fungal techniques, and histology. The survey was open from March 2018 
Table 2. Global survey results by specialty

\begin{tabular}{lcc}
\hline Global survey results & Dermatology & Family physician \\
\hline Median of correct diagnoses $(n ; 95 \% \mathrm{CI})$ & $10(9.31-10.76)$ & $6.2(5.74-6.65)$ \\
Minimum value & 2 & 0 \\
Maximum value & 14 & 11 \\
Median of unnecessary treatments $(n ; 95 \% \mathrm{CI})$ & $2(1.90-2.93)$ & $4(3.49-4.37)$ \\
Minimum value & 0 & 0 \\
Maximum value & 8 & 10 \\
\hline
\end{tabular}

Fig. 1. Percentage of correct diagnosis by

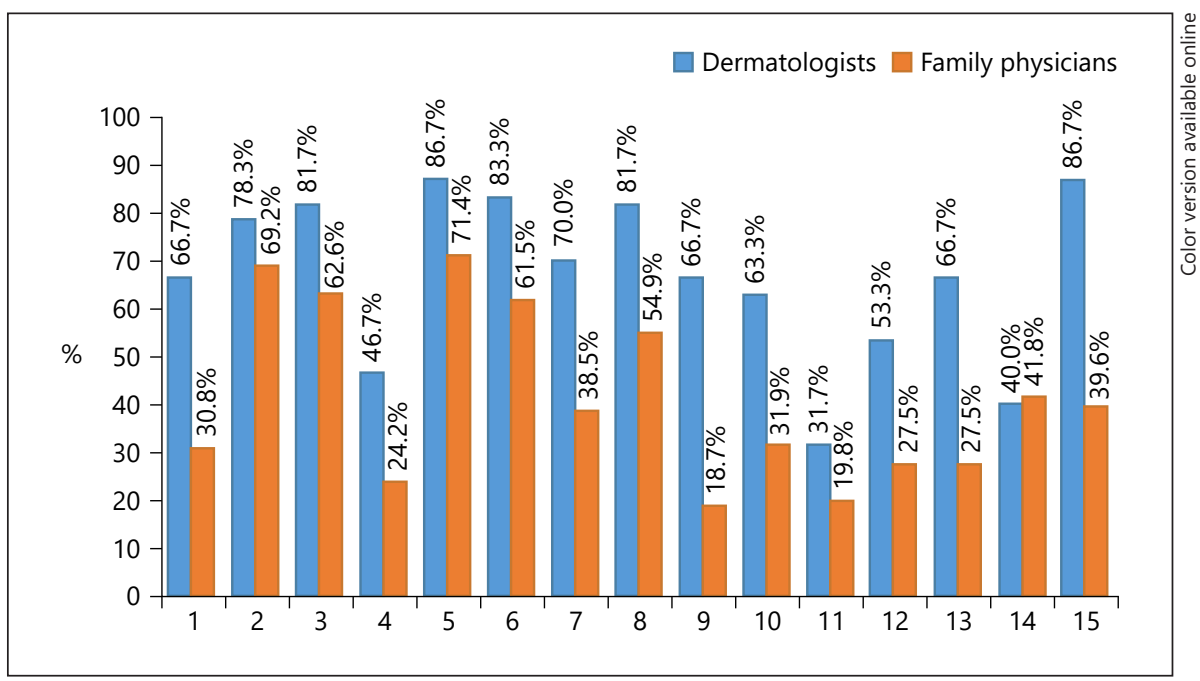
specialty and clinical case.

to December 2018. Assessment of survey results was done as follows: a diagnosis was deemed correct if in agreement with the confirmed diagnosis documented at our department; a treatment strategy was deemed unnecessary if it ultimately resulted in unwarranted treatment in accordance with the patient diagnosis (and/or initiated in the absence of prior consideration of mycological test results).

Statistical analysis was performed using Statistical Package for Social Sciences (SPSS) version 23.0. Descriptive statistics were provided using median (range) for normally and non-parametrically distributed numerical values, respectively. Percentages were used for categorical variables. Missing values were not included in calculation of percentages. Comparisons of numerical variables were analyzed with Q-Q Normal, Q-Q Normal without Trend, and the Shapiro-Wilk test having rejected normality. $\chi^{2}$, Mann-Whitney, or Fisher exact test were used for differences between categorical variables. $p$ values $<0.05$ were considered to be statistically significant.

\section{Results}

One hundred fifty-one responses were obtained, 60 (39.7\%) from dermatologists and 91 (60.3\%) from family physicians. Of the responders, 99 were female
(65.6\%) and 52 were males (34.4\%). Considering the groups independently, $60 \%$ of the dermatologists and $69.2 \%$ of the family physicians were female. From the responders, 88 were staff physicians $(58.3 \%)$, while 63 were residents (41.7\%). Dermatologists had a median age of 35.5 years (range 25-70 year) and family physicians 32 years (range 26-64 year). A total of $56 \%$ of the dermatologists and $92 \%$ of the family physicians mentioned working mostly or exclusively in the public healthcare system. Only a minority of dermatologists $(6.7 \%)$ dedicated less than $25 \%$ of their time to general dermatology (Table 1).

Considering the use of mycology diagnostic techniques in daily practice, $98.3 \%$ of dermatologists mentioned usually requesting fungal testing at their local institution or outside, while this percentage was significantly lower $(50.5 \%)$ among family physicians $(p<$ 0.001). Dermatologists mentioned an easier access to mycology exams, with $70 \%$ having access to direct microscopy and fungal culture at their local institution. On 
the other hand, a majority of family physicians (49.5\%) denied access to these. Family physicians who asked for testing mostly (46.2\%) did so, outside their place of work.

Considering global survey results, participants from the dermatology group had a median of 10 correct diagnoses, 6 for the family physicians $(p<0.001)$. The highest total number of correct diagnoses observed was 14 and 11 in the dermatologist and family physician group, and the lowest 2 and 0 , respectively (Table 2). Furthermore, 65\% of dermatologists would have their diagnosis ultimately confirmed in more than 8 cases, while the same was true for only $5.5 \%$ of family physicians shown in Figure $1(p<$ $0.001)$.

The choice of workup/treatment strategy, for each case, would result in unnecessary treatment in a median of 2 cases, for dermatologists, and 4 cases, for family physicians. The maximum number of unnecessary treatments that would have been started was 8 and 10 for dermatologists and family physicians, respectively, and the minimum was 0 in both groups. This last assertion was statistically significant $(p<0.001)$.

\section{Discussion}

We believe our sample of responders was heterogeneous and reflected the reality of our country. We obtained a good sample proportion of staff physicians versus residents, as well as dermatologists versus family physicians. Both groups were of a young adult age, which may be justified by the fact that younger physicians are more open to participating in an online survey methodology. The reality of health in our country has shown a trend in reducing the number of dermatologists working exclusively in the public sector. This trend is observed in our study, with only $56 \%$ of dermatologists working mostly or exclusively in public hospitals.

Regarding the request of mycology diagnostic techniques, the majority of dermatologists (98.3\%) request these in their daily practice in order to assess nail disease. In Portugal, mycology techniques are a training requirement in dermatology residency, a reason that can justify this higher percentage comparing to other studies [4]. Recent studies confirm the tendency of dermatologists to request mycological exams in a high percentage (91\%) [4]. However, the confirmation rate is lower in older reports such as the 1996 survey of Canadian Ds (69\%), 2005 EUROO study (39.6\%), and 2007 USA study (63.6\%) [57]. In the future, the incorporation of training in direct microscopy along with other tests which can be deployed in the clinic should be part of residency requirements for dermatologists [4]. Moreover, the majority of dermatologists $(70 \%)$ claimed to have easy access to direct microscopy and fungal culture at their institution, prompting their usage on a daily basis.

Approximately half of family physicians in our study did not diagnostically confirm onychomycosis. This similar percentage was found in another study [7]. It has been suggested that this may be because of issues related to time and procedure-related comfort [5-7]. Only a low percentage (3.4\%) of family physicians in the EUROO study reported requesting mycological exams [6]. These low percentages can be justified by the fact that this group has no training in the area of mycology, has more limitations in the access to the mycology techniques, and does not know how to collect mycological samples, or perhaps even interpret test results [7].

Regarding diagnostic familiarity, the median of correct diagnoses by the dermatologist group was higher $(10 / 15)$ than the family physicians (6/15). This reflects the training that dermatologists have through their residency and their daily practice. We could observe that when the cases were clinically more difficult, dermatologists were able to have higher scores. Moreover, it may be that family physicians are also less aware that other systemic, cutaneous, and/or biomechanical causes can lead to nail dystrophy that resembles onychomycosis [7].

Considering overall treatment strategy, we could observe that dermatologists were more conservative and tended to treat only when they were more certain of the diagnosis. Most dermatologists would wait for mycology test results before starting treatment. On the other hand, family physicians were more prone to prescribe unnecessary treatment in the event of an erroneous, clinically based diagnosis. This could have been related to lesser diagnostic acuity, a tendency to more often consider the diagnosis of onychomycosis and a decision to do without pre-treatment fungal testing. It is important to consider that prescribing unnecessary systemic antifungals has important consequences with potential side effects and frequent drug interactions.

We considered the following limitations to this study. Participants were requested to voluntarily participate; hence, a higher response rate could have been obtained. Selection bias was likely present; younger physicians and physicians who were more interested in nail disease related-issues were probably more likely to participate. 


\section{Conclusion}

This study provides important data regarding the request of mycology diagnostic techniques by dermatologists and family physicians in their daily current practice. It confirms that dermatologists consider it important to request for mycological investigation, especially prior treatment, having easier access to mycology exams at their institutions. Dermatologists are more familiarized with nail disease diagnosis with higher correct diagnosis than family physicians and tend to start treatment only when they are more certain of the diagnosis.

These findings also have training implications for dermatologists and family physicians. Training in mycology diagnostic techniques is relevant as a residency requirement for dermatologists. Family medicine residency programs should include specific training regarding nail disease diagnosis due to high prevalence of these conditions and global public health considerations regarding referral, workup, and treatment prescription.

\section{Statement of Ethics}

Not required for this study because there was no involvement of animals or experiences in humans.

\section{Conflict of Interest Statement}

The authors have no conflicts of interest to declare.

\section{Funding Sources}

No funding sources.

\section{Author Contributions}

Ana Filipe Monteiro - responsible for the conception and design of the work; acquisition, analysis, and interpretation of data for the work; drafting the work and revising it critically for important intellectual content; final approval of the version to be published; and agreement to be accountable for all aspects of the work in ensuring that questions related to the accuracy or integrity of any part of the work are appropriately investigated and resolved. Rita Pinheiro - responsible for the acquisition and analysis of data for the work, revising it for important intellectual content, approval of the version to be published, and agreement to be accountable for all aspects of the work in ensuring that questions related to the accuracy or integrity of any part of the work are appropriately investigated and resolved. Célia Galhardas - responsible for the acquisition, analysis and interpretation of the data for the work, drafting the work, approval of the version to be published, and agreement to be accountable for all aspects of the work in ensuring that questions related to the accuracy or integrity of any part of the work are appropriately investigated and resolved. André Lencastre - responsible for the conception and design of the work, acquisition, analysis and interpretation of data for the work, drafting the work and revising it critically for important intellectual content, final approval of the version to be published, and agreement to be accountable for all aspects of the work in ensuring that questions related to the accuracy or integrity of any part of the work are appropriately investigated and resolved.

\section{References}

1 Biesbroeck LK, Fleckman P. Nail disease for the primary care provider. Med Clin North Am. 2015;99(6):1213-26.

2 Gupta AK, Versteeg SG, Shear NH. Onychomycosis in the 21st century: an update on diagnosis, epidemiology, and treatment. J Cutan Med Surg. 2017;21(6):525-39

3 Ameen M, Lear JT, Madan V, Mohd Mustapa MF, Richardson M. British Association of Dermatologists' guidelines for the management of onychomycosis 2014. Br J Dermatol. 2014;171(5):937-58.
4 Saunte DML, Piraccini BM, Sergeev AY, Prohić A, Sigurgeirsson B, Rodríguez-Cerdeira C, et al. A survey among dermatologists: diagnostics of superficial fungal infections: what is used and what is needed to initiate therapy and assess efficacy? J Eur Acad Dermatology Venereol. 2018;33(2):421-7.

5 Gupta AK, Shear NH. A questionnaire study on the management of onychomycosis: a Canadian perspective. Int J Dermatol. 1998; 37(6):457-60
6 Effendy I, Lecha M, Feuilhade de Chauvin M, Di Chiacchio N, Baran R; European Onychomycosis Observatory. Epidemiology and clinical classification of onychomycosis. J Eur Acad Dermatology Venereol. 2005;19 (Suppl 1):8-12.

7 Koshnick RL, Lilly KK, Clair KS, Finnegan MT, Warshaw EM. Use of diagnostic tests by dermatologists, podiatrists and family practitioners in the United States: pilot data from a cross-sectional survey. Mycoses. 2007;50(6): 463-9. 